\title{
Severe Liver Toxicity in a Lung Cancer Patient Treated with Erlotinib: A Case Report and Literature Review
}

\section{Yi-Chun Lai ${ }^{1,2,4 *}$ and Jiun-I Lai ${ }^{2,3}$}

${ }^{1}$ Division of Chest Medicine, Department of Internal Medicine, National Yang-Ming University Hospital, Yilan, Taiwan

${ }^{2}$ School of Medicine, National Yang-Ming University, Taipei, Taiwan

${ }^{3}$ Department of Cell Molecular Biology, The Scripps Research Institute, USA

${ }^{4}$ Institute of Hospital and Health Care Administration, National Yang-Ming University, Taipei, Taiwan

\begin{abstract}
Background: Epidermal growth factor receptor (EGFR) tyrosine-kinase inhibitors (TKIs) constitute the standard therapy for advanced stage non-small cell lung cancer. Compared with systemic chemotherapy, TKI based treatment has fewer adverse effects.
\end{abstract}

Patients and methods: We present a lung cancer patient with erlotinib associated severe liver toxicity and perform a systemic review on published studies that report EGFR-TKI-associated liver toxicity. We conducted a systemic search in Medline, PubMed, and Google for studies that were accessible.

Results: A total of 18 papers was found from which we analysed 16 studies with a total of 30 patients. This is the largest case numbers literature review. In these studies, abnormal liver function levels were reported around $75.4 \pm 135.2$ days after initiating EGFR- TKI treatment. Six patients $(20 \%)$ died during the study period $(23.8 \pm 22.5$ days after EGFR-TKI use) who were more likely to be Male, to use erlotinib, to receive EGFR- TKI as non-first line therapy, and to have liver metastasis.

Conclusion: From our analysis, male, erlotinib use, EGFR-TKI for second line or later use, and initial liver metastasis were associated with higher mortality after EGFR-TKI-associated liver toxicity. The underlying mechanism is unknown, and further studies are required to validate these results.

Keywords: Epidermal growth factor receptor; Erlotinib; Hepatitis; Non-small cell lung cancer; Tyrosine-kinase inhibitor

\section{Introduction}

Erlotinib and gefitinib are orally taken, epidermal growth factor receptor (EGFR) tyrosine-kinase inhibitors (TKIs). Gefitinib was reported to increase progression free survival in advanced nonsmall cell lung cancer (NSCLC) with EGFR mutation [1,2]. Gefitinib was approved by US Food and Drug Administration (FDA) in 2003 for unresected lung cancer [3], and erlotinib received FDA approval in 2007 for the same indication [4]. The common adverse effects of EGFR-TKI included diarrhoea [2,5] and skin rash [6,7], although this seldom results in drug discontinuation [8]. The incidence of gefitinib associated liver toxicity (Grade 3 or 4, Common Terminology Criteria for Adverse Events 3.0) was around 9.4\% to $26.3 \%[1,2,9]$. However, erlotinib associated liver toxicity (Grade 3 or 4) [10] was less than 5\% [11], and the risk of liver function impairment is less than gefitinib. Under the dosage of $150 \mathrm{mg} /$ day in advanced NSCLC treated with erlotinib, no abnormal liver function impairment was reported [11-14]. There is no effective treatment for EGFR-TKI-associated liver toxicity, only discontinuation of the drug. However, we did not know what kind of patients will suffer liver toxicity with EGFR-TKI use till now.

Several case reports have described EGFR-TKI-associated liver toxicity, but most of the reports have a small sample size or are only a case report. We still do not understand the liver toxicity about EGFR-TKI in the real world. Herein, we present a case with erlotinib associated liver toxicity in a NSCLC, adenocarcinoma with positive EGFR mutation. In addition, we perform a review on all published case reports on EGFR-TKI-associated liver toxicity. This is the largest case series review of EGFR-TKI-associated liver toxicity.

\section{Materials and Methods}

\section{One case presentation}

A 64 years old male who was a heavy smoker presented to our hospital and was diagnosed with NSCLC, adenocarcinoma, cT3N2M1b, stage IV with multiple brain and solitary liver metastases on Nov $23^{\text {rd }}$, 2013. Endobronchial biopsy over the lung mass at right lower lobe was sent to pathology and revealed adenocarcinoma. Further genomic analysis confirmed the presence of EGFR exon 21 L858R point mutation. Erlotinib $150 \mathrm{mg}$ orally once daily was prescribed for first line therapy since Jan $8^{\text {th }}, 2014$. This patient had no underlying hepatitis B or $\mathrm{C}$, and no previous liver function impairment history before starting treatment. After 7 days of erlotinib use, baseline laboratory (lab) data before start of erlotinib revealed creatine $(\mathrm{Cr}), 1.33 \mathrm{mg} / \mathrm{dL}$, and normal liver function levels with aspartate aminotransferase (AST)/alanine aminotransferase (ALT), 20/41 U/L, total bilirubin, $0.98 \mathrm{mg} / \mathrm{dL}$.

On the twentieth day of erlotinib therapy, he complained about general weakness and poor appetite. The lab data showed $\mathrm{Cr}, 0.9 \mathrm{mg} / \mathrm{dL}$,

*Corresponding author: Lai YC, Division of Chest Medicine, Department of Internal Medicine, School of Medicine, National Yang-Ming University Hospital, Institute of Hospital and Health Care Administration, No. 152, Xin-Min Road, Yilan, Taiwan, 26042, Tel: +886-3-932-5192 ext. 1620; Fax: +886-3-935-1838; E-mail: mailto:toto881049@yahoo.com.tw

Received September 25, 2016; Accepted November 23, 2016; Published November 25,2016

Citation: Lai Y, Lai J (2016) Severe Liver Toxicity in a Lung Cancer Patient Treated with Erlotinib: A Case Report and Literature Review. J Cancer Sci Ther 8: 268-273. doi: 10.4172/1948-5956.1000425

Copyright: @ 2016 Lai Y, et al. This is an open-access article distributed under the terms of the Creative Commons Attribution License, which permits unrestricted use, distribution, and reproduction in any medium, provided the original author and source are credited. 
AST/ ALT, 342/738 U/L, total/direct bilirubin, 3.83/3.08 mg/dL, alkaline phosphatase (alk-p)/ gamma-glutamyl transferase (GGT), 322/485 U/L. Erlotinib was discontinued immediately under the impression of drug associated liver toxicity. Chest and abdominal computerized tomography (CT) showed a $5 \times 5 \times 4 \mathrm{~cm}$ solid mass over right lower lung, with tumour size regression and stationary liver metastasis lesion $(1 \times 1 \mathrm{~cm})$. The patient denied analgesics, Chinese herbs, and other overthe-counter drugs use. He was admitted for treatment of liver function impairment. After initial treatment with hydration, discontinuation of erlotinib and close monitoring of liver enzymes, his liver function continued to deteriorate in the first week of admission. Total bilirubin reached its peak of $11.03 \mathrm{mg} / \mathrm{dL}$ after discontinuing erlotinib for 9 days. We re-examined if hepatitis A, B or C was present, but the results were all negative. A sonogram-guided liver biopsy was performed for evaluation of the underlying cause. Pathology from the biopsy displayed chronic inflammatory cells infiltration in the periportal area and ballooning degeneration in some hepatocytes. Drug-associated liver toxicity could not be excluded as a diagnosis. We continued with our current treatment and limited unnecessary medication. Finally, the liver function levels recovered after discontinuing erlotinib for 28 days (Figure 1), and he was discharged. The patient is currently switched to first line chemotherapy with pemetrexed and cisplatin for advanced lung adenocarcinoma. At the time of this manuscript, initial preparation (Jan, 2014), his liver function levels are still within normal range. Unfortunately, he passed away in May, 2014 because of disease progression.

\section{Literature Review}

We performed a search in the literature using combinations of keyword phrases as follows: erlotinib/gefitinib related/induced hepatitis, abnormal liver function, liver toxicity, and lung cancer, in Medline, PubMed and Google, with our search limited to English publications. A total of 18 papers were found [13,15-31]. Two studies were discarded resulting in 16 manuscripts enrolled (Table 1 ) including 12 case reports [13,15-18,20,21,24,26,29-31], 1 literature review [19], 1 letter to the editors [22] and two case series [23,25]. Of the two studies omitted, one was a meta-analysis [27] and one was a case report [28] which did not reveal clinical detail laboratory data for our further analysis.
We collected the patients' clinical characteristics, EGFR-TKI type, use duration, and laboratory data reported in the manuscript. We wanted to analyze the severity and mortality about the EGFR-TKIassociated liver toxicity.

\section{Statistical Analysis}

The continuous variable is presented as mean \pm standard deviation (SD) and binary variables are presented with percentage, such as age, EGFR TKIs use period, etc. The difference between erlotinib and gefitinib group were compared using chi square or Fisher exact test. All statistics were performed using the SPSS 22.0 (SPSS Inc., Chicago, IL, USA).

\section{Results}

A total of 30 cases reported in our searches (including our case) of EGFR-TKI-associated liver toxicity was identified. Basic demographic characteristics are presented in Table 2. The mean age was $63.6 \pm 13.1$ years old, with 18 (60\%) female patients. Twenty-nine (93.5\%) patients had the pathology of adenocarcinoma and only one case reported non-small cell lung cancer. Sixteen $(53.3 \%)$ patients had positive EGFR mutation, while in 12 cases $(40 \%)$ the mutation status was not mentioned in the manuscripts. Eighteen $(60 \%)$ patients were treated with gefitinib and others with erlotinib. Seventeen patients (56.7\%) were treated with EGFR-TKI as first line therapy. In addition, although $4(13.3 \%)$ patients had liver metastasis during EGFR- TKI treatment, none had a reported history of hepatitis B or C. Only less than 15\% of the patients had symptoms such as skin rash, nausea, or vomiting when abnormal liver function was present. Most physicians (28 cases, 93.3\%) discontinued EGFR-TKI at the initial presentation of abnormal liver function levels, and 3 cases (13.3\%) were treated with steroids. Finally, 19 patients $(63.4 \%)$ were switched to a different EGFR-TKI after liver function test normalized.

Almost all patients had nearly normal liver function test at the initial treatment with EGFR-TKI (Table 3). The time point of initial abnormal liver function was at $75.4 \pm 135.2$ days after starting EGFRTKI treatment. The peak liver enzyme levels were: AST, $632.7 \pm 780.8$

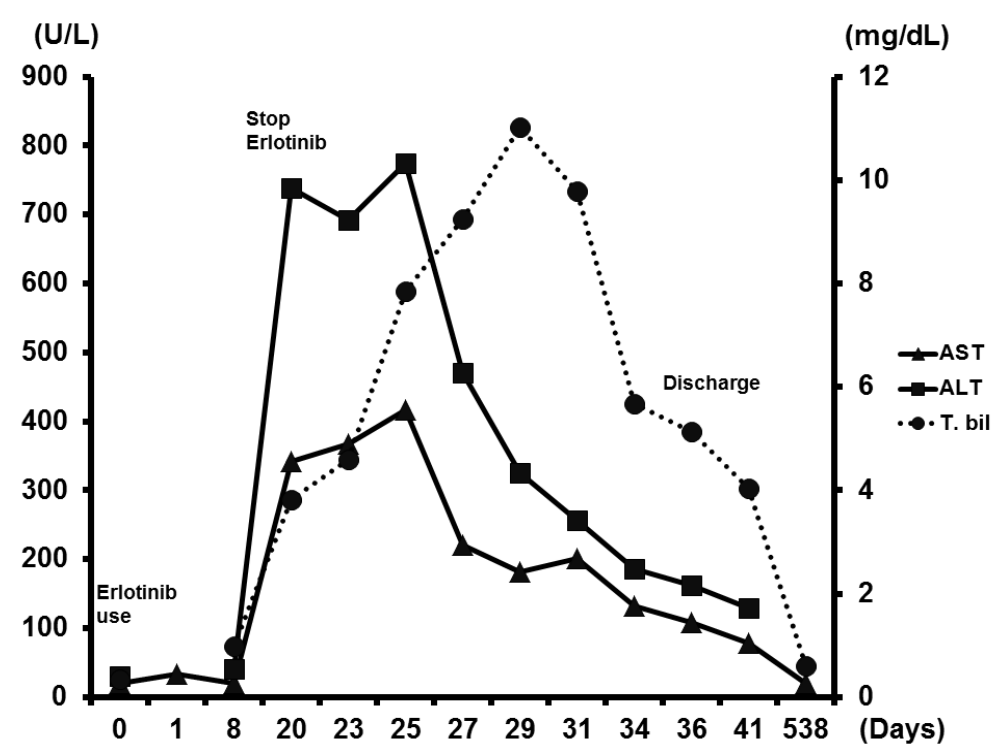

Figure 1: Result of the series liver function test in our patient during erlotinib treatment course and discontinuation of the drug. The liver function test recovered after about 40 days. AST: aspartate aminotransferase; ALT: alanine aminotransferase; L: liter; T. bil: total bilirubin; dL: decileter; mg: milligrams. 
Citation: Lai Y, Lai J (2016) Severe Liver Toxicity in a Lung Cancer Patient Treated with Erlotinib: A Case Report and Literature Review. J Cancer Sci Ther 8: 268-273. doi: 10.4172/1948-5956.1000425

\begin{tabular}{|c|c|c|c|c|c|c|c|c|c|}
\hline Cancer type & Age/Sex & $\begin{array}{l}\text { Initial AST/ALT } \\
\text { (mg/dL) }\end{array}$ & $\begin{array}{c}\text { Peak AST/ALT } \\
\text { (mg/dL) }\end{array}$ & $\begin{array}{l}\text { Initial T. bil } \\
\text { (U/L) }\end{array}$ & $\begin{array}{l}\text { Peak T. bil } \\
\text { (U/L) }\end{array}$ & Management & $\begin{array}{l}\text { Out } \\
\text { come }\end{array}$ & $\begin{array}{l}\text { Time to first } \\
\text { elevate LTF } \\
\text { (days) }\end{array}$ & $\begin{array}{l}\text { Time to } \\
\text { recover LFT } \\
\text { (days) }\end{array}$ \\
\hline Adenocarcinoma $^{15}$ & $53 / \mathrm{M}$ & - & $3511 / 884$ & 1.1 & 4.7 & hydration & Die & 10 & - \\
\hline $\mathrm{NSCLC}^{16}$ & $81 / \mathrm{M}$ & $34 / 28$ & $97 / 70$ & 1.2 & 13.6 & Stop drug & live & 14 & 10 \\
\hline Adenocarcinoma $^{17}$ & $67 / F$ & $25 / 30$ & $2476 / 2021$ & 0.8 & 15.9 & $\begin{array}{l}\text { Steroid, stop } \\
\text { drug }\end{array}$ & Die & 14 & - \\
\hline Adenocarcinoma ${ }^{18}$ & 77/F & - & $340 / 170$ & - & - & stop drug & live & 67 & 40 \\
\hline Adenocarcinoma $^{19}$ & $74 / \mathrm{M}$ & $13 / 12$ & $108 / 116$ & - & - & $\begin{array}{l}\text { Steroid, stop } \\
\text { drug }\end{array}$ & live & 22 & 4 \\
\hline Adenocarcinoma $^{20}$ & $64 / F$ & - & $592 / 617$ & - & - & stop drug & live & 35 & 23 \\
\hline Adenocarcinoma ${ }^{21}$ & $66 / F$ & $84 / 181$ & $1011 / 599$ & - & - & stop drug & live & 91 & 49 \\
\hline Adenocarcinoma $^{22}$ & $31 / \mathrm{M}$ & $53 / 53$ & $431 / 896$ & 0.9 & 6 & stop drug & Die & 2 & - \\
\hline Adenocarcinoma $^{22}$ & $58 / \mathrm{M}$ & $20 / 18$ & $800 / 745$ & 0.8 & 13 & stop drug & Die & 7 & - \\
\hline Adenocarcinoma $^{22}$ & $72 / \mathrm{M}$ & $38 / 49$ & $1250 / 1412$ & 0.7 & 3.3 & stop drug & Die & 6 & - \\
\hline Adenocarcinoma $^{23}$ & $50 / F$ & - & $63 / 155$ & - & - & stop drug & live & 22 & - \\
\hline Adenocarcinoma $^{23}$ & $51 / F$ & - & $159 / 280$ & - & - & stop drug & live & 175 & - \\
\hline Adenocarcinoma $^{23}$ & $61 / \mathrm{M}$ & - & $128 / 197$ & - & - & stop drug & live & 42 & - \\
\hline Adenocarcinoma $^{23}$ & $64 / F$ & - & $89 / 263$ & - & - & stop drug & live & 53 & - \\
\hline Adenocarcinoma $^{23}$ & $64 / F$ & - & $426 / 533$ & - & - & stop drug & live & 55 & - \\
\hline Adenocarcinoma $^{23}$ & $66 / F$ & - & $52 / 252$ & - & - & stop drug & live & 191 & - \\
\hline Adenocarcinoma $^{23}$ & $72 / \mathrm{M}$ & - & $231 / 342$ & - & - & stop drug & live & 35 & - \\
\hline Adenocarcinoma $^{23}$ & $76 / F$ & - & $62 / 113$ & - & - & stop drug & live & 50 & - \\
\hline Adenocarcinoma ${ }^{24}$ & $31 / \mathrm{F}$ & - & $972 / 3720$ & - & - & stop drug & live & 28 & 14 \\
\hline Adenocarcinoma $^{25}$ & $66 / F$ & - & $599 / 1053$ & - & - & stop drug & live & - & - \\
\hline Adenocarcinoma $^{25}$ & $63 / F$ & - & $226 / 519$ & - & - & stop drug & live & - & - \\
\hline Adenocarcinoma $^{25}$ & $53 / F$ & - & $765 / 1053$ & - & - & stop drug & live & 42 & - \\
\hline Adenocarcinoma $^{33}$ & $57 / F$ & - & $800 /-$ & - & - & $\begin{array}{l}\text { Steroid, stop } \\
\text { drug }\end{array}$ & live & 56 & 60 \\
\hline Adenocarcinoma $^{13}$ & $77 / \mathrm{M}$ & $18 / 22$ & $1400 / 1299$ & - & 1 & stop drug & Die & 12 & - \\
\hline Adenocarcinoma (our case) & $64 / \mathrm{M}$ & $20 / 41$ & $182 / 325$ & 0.98 & 11 & stop drug & Live & 20 & 60 \\
\hline Adenocarcinoma ${ }^{29}$ & $61 / F$ & - & $629 / 1130$ & - & - & stop drug & Live & 720 & 300 \\
\hline Adenocarcinoma $^{30}$ & $67 / F$ & - & $-/ 731$ & - & - & stop drug & Live & 120 & 120 \\
\hline Adenocarcinoma $^{30}$ & $83 / F$ & $-/ 124$ & $-/ 375$ & - & - & stop drug & Live & 60 & 100 \\
\hline Adenocarcinoma $^{31}$ & $52 / \mathrm{M}$ & - & $173 / 354$ & - & - & stop drug & live & 120 & 30 \\
\hline Adenocarcinoma $^{31}$ & $88 / \mathrm{M}$ & - & $143 / 287$ & - & - & stop drug & live & 42 & 35 \\
\hline
\end{tabular}

LFT: liver function test; AST: aspartate aminotransferase; ALT: alanine aminotransferase; L: liter; T. bil: total bilirubin; dL: decileter; M: male; F: female; NSCLC: non-small cell lung cancer

Table 1: Published cases of erlotinib-associated liver toxicity in lung cancer. Out-come, time to first elevate LFT (days).

\begin{tabular}{|c|c|}
\hline Characteristics & $\mathbf{N}(\%)$ unless otherwise indicated, total $=30$ \\
\hline Age in year, mean $\pm S D$ (range) & $63.6 \pm 13.1(31-81)$ \\
\hline Female & $18(60 \%)$ \\
\hline Cancer type (adenocarcinoma) & $29(93.5 \%)$ \\
\hline \multicolumn{2}{|c|}{ Stage } \\
\hline $\mathrm{Illb}$ & $3(10 \%)$ \\
\hline IV & $15(50 \%)$ \\
\hline unknown & $12(40 \%)$ \\
\hline \multicolumn{2}{|c|}{ EGFR mutation } \\
\hline positive & $16(53.3 \%)$ \\
\hline negative & $2(6.7 \%)$ \\
\hline unknown & $12(40 \%)$ \\
\hline \multicolumn{2}{|c|}{ TKI use } \\
\hline Gefitinib & $18(60 \%)$ \\
\hline Erlotinib & $12(40 \%)$ \\
\hline Smoke & $6(20 \%)$ \\
\hline \multicolumn{2}{|c|}{ ECOG } \\
\hline 0 & $4(13.3 \%)$ \\
\hline
\end{tabular}


Citation: Lai Y, Lai J (2016) Severe Liver Toxicity in a Lung Cancer Patient Treated with Erlotinib: A Case Report and Literature Review. J Cancer Sci Ther 8: 268-273. doi: 10.4172/1948-5956.1000425

\begin{tabular}{|c|c|}
\hline 1 & $3(10 \%)$ \\
\hline 2 & $3(10 \%)$ \\
\hline 3 & $6(20 \%)$ \\
\hline unknown & $14(46.7 \%)$ \\
\hline \multicolumn{2}{|c|}{ Treatment } \\
\hline First line & $17(56.7 \%)$ \\
\hline Second line & $3(10 \%)$ \\
\hline Third line & $2(6.7 \%)$ \\
\hline Unknown & $8(26.6 \%)$ \\
\hline Liver metastasis & $4(13.3 \%)$ \\
\hline Hepatitis history & $0(0 \%)$ \\
\hline \multicolumn{2}{|c|}{ Pre-treatment with TKI } \\
\hline Gefitinib & $6(20 \%)$ \\
\hline No & $24(80 \%)$ \\
\hline Pre-treatment with chemotherapy & $4(13.3 \%)$ \\
\hline \multicolumn{2}{|c|}{ Outcome } \\
\hline Die & $6(20 \%)$ \\
\hline Survival & $24(80 \%)$ \\
\hline \multicolumn{2}{|c|}{ Symptoms } \\
\hline Skin rash & $3(10 \%)$ \\
\hline Dyspnea & $3(10 \%)$ \\
\hline Nausea & $4(13.3 \%)$ \\
\hline Poor appetite & $3(13.3 \%)$ \\
\hline Abdominal pain & $2(6.7 \%)$ \\
\hline Fever & $1(3.3 \%)$ \\
\hline Stop EGFR-TKI & $28(93.3 \%)$ \\
\hline Treatment with steroid & $3(13.3 \%)$ \\
\hline Shift to another EGFR-TKI & $19(63.4 \%)$ \\
\hline
\end{tabular}

Table 2: Characteristics of the reported patients with EGFR-TK-associated liver toxicity.

\begin{tabular}{|c|c|c|}
\hline \multicolumn{2}{|c|}{ Clinical data } & Mean \pm SD \\
\hline \multirow{3}{*}{$\begin{array}{l}\text { Initial } \\
\text { treatment }\end{array}$} & AST (U/L) & $34 \pm 22.1$ \\
\hline & $\operatorname{ALT}(\mathrm{U} / \mathrm{L})$ & $55.8 \pm 54.3$ \\
\hline & T. bil (mg/dL) & $0.9 \pm 0.2$ \\
\hline \multirow{3}{*}{ Peak level } & AST (U/L) & $632.7 \pm 780.8$ \\
\hline & $\operatorname{ALT}(\mathrm{U} / \mathrm{L})$ & $705.8 \pm 742.5$ \\
\hline & T. bil (mg/dL) & $7.7 \pm 5.8$ \\
\hline \multicolumn{2}{|c|}{ Time to liver function impairment (days) } & $75.4 \pm 135.2$ \\
\hline \multicolumn{2}{|c|}{ Time to peak liver function impairment (days) } & $114.4 \pm 172.8$ \\
\hline \multicolumn{2}{|c|}{$\begin{array}{l}\text { Time to liver function recovery after } \\
\text { discontinuing EGFR-TKI (days) }(\mathrm{N}=7)\end{array}$} & $65.4 \pm 81.8$ \\
\hline \multicolumn{2}{|c|}{ Survival days $(\mathrm{N}=6)$} & $23.8 \pm 22.5$ \\
\hline
\end{tabular}

AST: Aspartate aminotransferase; ALT: Alanine aminotransferase; T. bil: Total bilirubin; SD: Standard deviation; EKFR-TKI: Epidermal growth factor receptortyrosine kinase inhibitor; N: Number

Table 3: Summary of liver function test in the reported cases.

U/L, ALT, $705.8 \pm 742.5 \mathrm{U} / \mathrm{L}$, total bilirubin, $7.7 \pm 5.8 \mathrm{mg} / \mathrm{dL}$. It took $65.4 \pm 81.8$ days for liver function to recover after discontinuing EGFRTKI. There were 6 patients (20\%) who ultimately expired. In these 6 patients, the length of survival was $23.8 \pm 22.5$ days after EGFR-TKI use.

We found dead patients tended to be male ( $\mathrm{p}=0.02)$, to use erlotinib $(\mathrm{p}=0.001)$, to receive EGFR-TKI as non-first-line therapy $(\mathrm{p}=0.008)$, and to have liver metastasis ( $\mathrm{p}=0.003$ ) (Table 4 ).

\section{Discussion}

First, we present an advance lung adenocarcinoma patient treated with erlotinib with drug-induced liver toxicity. Then, we reviewed a total of 30 cases reported in the internet about EGFR-TKI-induced liver toxicity, with 6 fatal cases. It took $65.4 \pm 81.8$ days for liver function to recover after discontinuing EGFR-TKI. In the 6 fatal cases, length of survival was $23.8 \pm 22.5$ days after EGFR-TKI use. We found the non-survival patients tended to be male, to use erlotinib, to receive EGFR-TKI as non-first-line therapy, and have liver metastasis. To our knowledge, this is the first and largest of lung cancer cases to be systemically reviewed concerning EGFR-TKI -associated hepatitis.

Liver function impairment was a rare adverse effect of EGFR-TKI in phase III clinical trials [4]. Clinically, it is common to observe mild elevation of liver function tests in lung cancer patients with EGFRTKI use. However, it is unclear why some patients using EGFR-TKI suffer from fatal hepatic toxicity, but while others seem to tolerate TKI well. CYP3A4 polymorphisms have been associated with liver toxicity in TKI use [32], which slow the rate of EGFR-TKI metabolism and ultimately result in higher circulating drug levels [22], although the exact mechanism is yet to be determined. Currently, there is no standard examination for prediction of EGFR-TKI induced hepatitis, and predisposing risk factors are unclear. It must be noted that our study provides a hint of associated risk factors, but a causal effect can only be established firmly by a prospectively designed clinical trial.

Treatment-wise, there is no specific treatment other than discontinuation of TKI and close monitoring of liver function levels. One solitary case report [19] presented successful treatment with high dose methylprednisolone, but this has yet to be validated by more studies. Close observation and interruption of suspected drugs and supportive care remain the current treatment.

Our study is subject to several limitations. First, publication bias should be considered due to our search criteria limiting the results to online publication full text. It is likely that less severe cases might not be 


\begin{tabular}{|c|c|c|c|}
\hline & Survive $(n=24)(\%)$ & Die $(n=6)(\%)$ & $P$ value \\
\hline \multicolumn{3}{|c|}{ Sex } & \multirow[t]{3}{*}{0.02} \\
\hline Male & $7(29.2)$ & $5(83.3)$ & \\
\hline Female & $17(70.8)$ & $1(16.7)$ & \\
\hline Age (years old) & $64.6 \pm 12.3$ & $59.7 \pm 6.8$ & 0.42 \\
\hline \multicolumn{3}{|c|}{ Stage } & 0.56 \\
\hline IIIb & $1(8.3)$ & $2(33.3)$ & \\
\hline IV & $11(91.7)$ & $4(66.7)$ & \\
\hline \multicolumn{3}{|c|}{ EGFR mutation } & 0.11 \\
\hline Positive & $15(93.7)$ & $1(50)$ & \\
\hline Negative & $1(6.3)$ & $1(50)$ & \\
\hline \multicolumn{3}{|c|}{ EGFR-TKI type } & 0.001 \\
\hline Erlotinib & $6(25)$ & $6(100)$ & \\
\hline Gefitinib & $18(75)$ & $0(0)$ & \\
\hline \multicolumn{3}{|c|}{ Smoke } & 0.30 \\
\hline Yes & $6(25)$ & $0(0)$ & \\
\hline No & $18(75)$ & $6(100)$ & \\
\hline \multicolumn{3}{|c|}{ ECOG } & 0.26 \\
\hline 0 & $3(25)$ & $1(25)$ & \\
\hline 1 & $3(25)$ & $0(0)$ & \\
\hline 2 & $3(25)$ & $0(0)$ & \\
\hline 3 & $3(25)$ & $3(75)$ & \\
\hline \multicolumn{3}{|c|}{ Treatment } & 0.008 \\
\hline First line & $15(93.8)$ & $2(33.3)$ & \\
\hline Second line & $1(6.3)$ & $2(33.3)$ & \\
\hline Third line & $0(0)$ & $2(33.3)$ & \\
\hline \multicolumn{3}{|c|}{ Liver metastasis } & 0.003 \\
\hline Yes & $1(4.2)$ & $3(50)$ & \\
\hline No & $23(95.8)$ & $3(50)$ & \\
\hline \multicolumn{3}{|c|}{ Pre-treatment with TKI } & 0.82 \\
\hline Gefinitib & $5(20.8)$ & $1(16.7)$ & \\
\hline No & $19(79.2)$ & $5(83.3)$ & \\
\hline \multicolumn{3}{|c|}{ Pre-treatment with chemotherapy } & 0.003 \\
\hline Yes & $1(4.2)$ & $3(50)$ & \\
\hline No & $23(95.8)$ & $3(50)$ & \\
\hline \multicolumn{3}{|c|}{ Treatment with steroid } & 0.54 \\
\hline Yes & $2(8.3)$ & $1(16.7)$ & \\
\hline No & $22(91.7)$ & $5(83.3)$ & \\
\hline \multicolumn{3}{|c|}{ Stop EGFR-TKI } & 0.003 \\
\hline Yes & $24(100)$ & $4(66.7)$ & \\
\hline No & $0(0)$ & $2(33.3)$ & \\
\hline
\end{tabular}

EKFR-TKI: epidermal growth factor receptor-tyrosine kinase inhibitor; N: number; ECOG: Eastern cooperative oncology group

Table 4: Outcome of EGFR-TKI-associated liver toxicity per patient characteristics.

reported by physician, and it is possible that not all cases will be reported since there is currently no registry for TKI related hepatitis. Otherwise, these case reports are diagnosed with TKI-induced liver toxicity at a certain severity level. The cases we collected still had representative and reference value. Second, some crucial patient characteristics are absent in some of the reports, such as smoking condition or performance status. Therefore, these factors that we have insufficient data on would be omitted from analysis. Third, since our study is a retrospective literature analysis, it lacks the evidence level of a prospective trial. Fourth, patients receiving second- or later-line TKI treatment may have a worse outcome than patients receiving first-line TKI treatment. Initial liver metastasis also is known to be a bad prognostic factor. Nevertheless, our study provides insights into possible adverse effects in lung cancer patients using TKI and can serve as important information for clinicians prescribing TKIs to patients, especially raising concern in patients with predisposed liver impairment.
In conclusion, although EGFR-TKIs offer proven efficacy and are usually well tolerated in lung cancer patients, prescribing physicians should be alert to hepatitis as a possible side effect, and liver enzymes must be followed closely in high risk patients. Per our analysis of 30 cases, in case of EGFR-TKI-associated liver toxicity as defined in this review, the use of erlotinib, being male and having initial liver metastasis were found to have a negative prognostic value and should be kept in mind when initiating TKI treatment courses.

\section{References}

1. Maemondo M, Inoue A, Kobayashi K, Sugawara S, Oizumi S, et al. (2010) Gefitinib or chemotherapy for non-small-cell lung cancer with mutated EGFR. New Engl j med 362: 2380-2388.

2. Mitsudomi T, Morita S, Yatabe Y, Negoro S, Okamoto I, et al. (2010) Gefitinib versus cisplatin plus docetaxel in patients with non-small-cell lung cancer harbouring mutations of the epidermal growth factor receptor (WJTOG3405) An open label, randomised phase 3 trial. Lancet Oncol 11: 121-128.

3. Kris MG, Natale RB, Herbst RS, Lynch TJ Jr, Prager D, et al. (2003) Efficacy of gefitinib, an inhibitor of the epidermal growth factor receptor tyrosine kinase, in symptomatic patients with non-small cell lung cancer: a randomized trial. JAMA 290: $2149-2158$.

4. Shepherd FA, Rodrigues Pereira J, Ciuleanu T, Tan EH, Hirsh V, et al. (2005) Erlotinib in previously treated non-small-cell lung cancer. N Engl J Med 353: 123-132.

5. Rosell R, Carcereny E, Gervais R, Vergnenegre A, Massuti B, et al. (2012) Erlotinib versus standard chemotherapy as first-line treatment for European patients with advanced EGFR mutation-positive non-small-cell lung cancer (EURTAC): a multicentre, open-label, randomised phase 3 trial. Lancet Onco 13: $239-246$.

6. Perez-Soler R, Delord JP, Halpern A, Kelly K, Krueger J, et al. (2005) HER1/ EGFR inhibitor-associated rash: future directions for management and investigation outcomes from the HER1/EGFR inhibitor rash management forum. The oncologist 10: 345-356.

7. Hirsh V (2011) Managing treatment-related adverse events associated with egfr tyrosine kinase inhibitors in advanced non-small-cell lung cancer. Curr oncol 18: 126-138.

8. Gridelli C, Bareschino MA, Schettino C, Rossi A, Maione P, et al. (2007) Erlotinib in non-small cell lung cancer treatment: current status and future development. Oncologist 12: 840-849.

9. Mok TS, Wu YL, Thongprasert S, Yang CH, Chu DT, et al. (2009) Gefitinib or carboplatin-paclitaxel in pulmonary adenocarcinoma. N Engl J Med 361: 947-957.

10. $\mathrm{NCl}$ (2006) Common terminology criteria for adverse events v3.0 (CTCAE) Cancer therapy evaluation program, common terminology criteria for adverse events. NIH, USA.

11. Kubota K, Nishiwaki Y, Tamura T, Nakagawa K, Matsui K, et al. (2008) Efficacy and safety of erlotinib monotherapy for Japanese patients with advanced nonsmall cell lung cancer: A phase II study. J thorac oncol 3: 1439-1445.

12. Herbst RS, Prager D, Hermann R, Fehrenbacher L, Johnson BE, et al. (2005) TRIBUTE: A phase III trial of erlotinib hydrochloride (OSI-774) combined with carboplatin and paclitaxel chemotherapy in advanced non-small-cell lung cancer. J clin oncol 23: 5892-5899.

13. Pellegrinotti M, Fimognari FL, Franco A, Repetto L, Pastorelli R (2009) Erlotinibinduced hepatitis complicated by fatal lactic acidosis in an elderly man with lung cancer. Ann Pharmacother 43: 542-545.

14. Yoshioka H, Hotta K, Kiura K, Takigawa N, Hayashi H, et al. (2010) A phase I trial of erlotinib monotherapy in pre-treated patients with advanced non-smal cell lung cancer who do not possess active EGFR mutations: Okayama lung cancer study group trial 0705. J thorac oncol 5: 99-104.

15. Schacher-Kaufmann S, Pless M (2010) Acute fatal liver toxicity under Erlotinib Case Rep Oncol 3: 182-188.

16. Arora AK (2011) Erlotinib-induced hepatotoxicity-clinical presentation and successful management: A Case Report. J Clin Exp Hepatol 1: 38-40.

17. Liu W, Makrauer FL, Qamar AA, Jänne PA, Odze RD (2007) Fulminant hepatic failure secondary to erlotinib. Clin Gastroenterol Hepatol 5: 917-920. 
Citation: Lai Y, Lai J (2016) Severe Liver Toxicity in a Lung Cancer Patient Treated with Erlotinib: A Case Report and Literature Review. J Cancer Sci Ther 8: 268-273. doi: 10.4172/1948-5956.1000425

18. Liu CC, Chen YM (2009) Recovery from gefitinib-induced pneumonitis and hepatitis after changing to erlotinib treatment. $J$ Chinese Oncol Soc 25: $439-445$.

19. Lai YC, Lin PC, Lai JI, Hsu SY, Kuo LC, et al. (2011) Successful treatment of erlotinib-induced acute hepatitis and acute interstitial pneumonitis with highdose corticosteroid: A case report and literature review. Int j clin pharmacol ther 49: 461-466.

20. Kunimasa K, Yoshioka H, Iwasaku M, Nishiyama A, Korogi Y, et al. (2012) Successful treatment of non-small cell lung cancer with gefitinib after severe erlotinib-related hepatotoxicity. Int med 51: 431-434.

21. Takeda M, Okamoto I, Fukuoka M, Nakagawa K (2010) Successful treatment with erlotinib after gefitinib-related severe hepatotoxicity. J clin oncol 28: e273-274.

22. Huang YS, An SJ, Chen ZH, Wu YL (2009) Three cases of severe hepatic impairment caused by erlotinib. Br J clin pharmacol 68: 464-467.

23. Yano Y, Namba Y, Mori M, Nakazawa Y, Nashi A, et al. (2012) Treatment of non-small-cell lung cancer with Erlotinib following Gefitinib-induced hepatotoxicity: Review of 8 Clinical Cases. Lung Canc Int 354657.

24. Nakatomi K, Nakamura Y, Tetsuya I, Kohno S (2011) Treatment with gefitinib after erlotinib-induced liver injury: a case report. J Med Case Rep 5: 593.

25. Takeda M, Okamoto I, Tsurutani J, Oiso N, Kawada A, et al. (2012) Clinical impact of switching to a second EGFR-TKI after a severe AE related to a first EGFR-TKI in EGFR-mutated NSCLC. Jpn J Clin Oncol 42: 528-533.
26. Ho C, Davis J, Anderson F, Bebb G, Murray N (2005) Side effects related to cancer treatment: CASE 1. Hepatitis following treatment with gefitinib. J clin oncol 23: 8531-8533.

27. Teo YL, Ho HK, Chan A (2013) Risk of tyrosine kinase inhibitors-induced hepatotoxicity in cancer patients: a meta-analysis. Canc treat rev 39: 199-206.

28. Shinji Teramoto HY, Yasuyoshi Ouchi (2003) Reason to stop treatment with gefitinib in patients with inoperable lung cancer. The Lancet 361: 1993.

29. Chen X, Pan Y, Zhang S, Chen D, Yang S, et al. (2014) Rechallenge with gefitinib following severe drug-induced hepatotoxicity in a patient with advanced non-small cell lung cancer: A case report and literature review. Oncol lett 7: 878-880.

30. Kijima T, Shimizu T, Nonen S, Furukawa M, Otani $Y$, et al. (2011) Safe and successful treatment with erlotinib after gefitinib-induced hepatotoxicity: difference in metabolism as a possible mechanism. J clin oncol 29: e588-590.

31. Ku GY, Chopra A, de Lopes GL Jr (2010) Successful treatment of two lung cancer patients with erlotinib following gefitinib-induced hepatotoxicity. Lung Canc 70: 223-225.

32. Rudin CM, Liu W, Desai A, Karrison T, Jiang X, et al. (2008) Pharmacogenomic and pharmacokinetic determinants of erlotinib toxicity. J Clin Oncol 26: 1119-1127.

33. Arshad M, Seiter K, Bilaniuk J, Qureshi A, Patil A, et al. (2005) Side effects related to cancer treatment: CASE 2 . Splenic rupture following pegfilgrastim. J Clin Oncol 23: 8533-8534. 